\title{
Satellite Image Retrieval using Modified Block Truncation Coding and Kekre Transform Patterns
}

\author{
A R Sawant \\ M. E. EXTC \\ TCET, Kandivali \\ Mumbai-400 101
}

\author{
Dr. Vinayak A Bharadi, \\ Bijith Markarkandy \\ IT Department, TCET, Kandivali, \\ Mumbai-400 101
}

\author{
Dr. H B Kekre \\ Computer Department \\ MPSTME, NMIMS \\ University,Mumbai-28
}

\begin{abstract}
Satellite images and Content Based Image Retrieval are interesting topics of research since years. Specifically, CBIR is on developing technologies for bridging the semantic gap that currently prevents wide-deployment of image content-based search engines. Image search engines currently in use are mostly rely on human generated data, such as text. Annotation of an image is totally depend on the person's perception who is going to store it into database. It is time-consuming as well as error prone. Therefore search engine using text input results in various nonrelevant images. To overcome drawbacks of text based image retrieval, Content based image retrieval is introduced where retrieval of images is totally depend on the features of images. Mostly, content-based methods are based on low-level descriptions, while high-level or semantic descriptions are beyond current capabilities. In this paper, we will try to implement the technique to fill this gap. This technique can eventually be extended to allow for content-based similarity type of search, to find out different query blocks from satellite image. We will fire an object as a query and most similar blocks will get retrieved. This will help out to trace particular object from large satellite image.
\end{abstract}

Keywords--Image retrieval; CBIR; MBTC; Kekre's pattern; Satellite image retrieval.

\section{INTRODUCTION}

Retrieval is the wide topic of research from the decades, because it is a challenge to reduce the semantic gap Basically retrieval of data means to get desired data from the database. It may image, text, audio or video as per requirement of user. The basic types of retrievals are mentioned in figure(1).

From ages images have been the mode of communication for human being. Today we are able to generate, store, transmit and share enormous amount of data because of the exhaustive growth of Information and Communication Technology. Much of this information is multimedia in nature, which consists of digital images, video, audio, graphics, and text data [1], [2]. But all that information is only useful if one can access it efficiently. This does not only mean fast access from a storage management point of view but also means that one should be able to find the desired information without scanning all information manually.

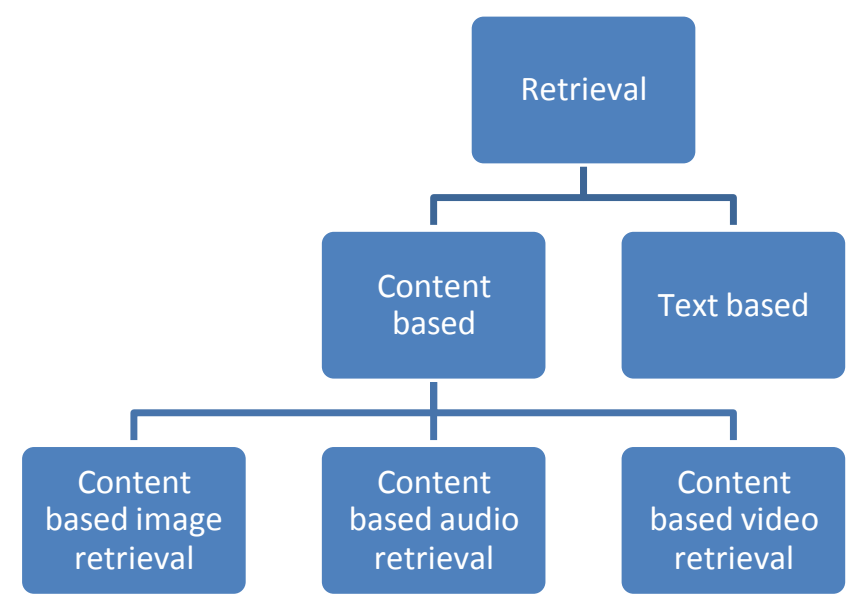

Fig.1. Types of retrieval

Previous method used for image retrieval is Text based image retrieval. The advantage of textual indexing of image is that it can provide user with key word searching, catalogue browsing and even with query interface. But the major drawback of text based image retrieval are, annotation depends on the person who adds it. [4] Due to all these drawbacks, Content Based Image Retrieval is introduced.

Here we will be dealing with three features of images shape, texture and color. 


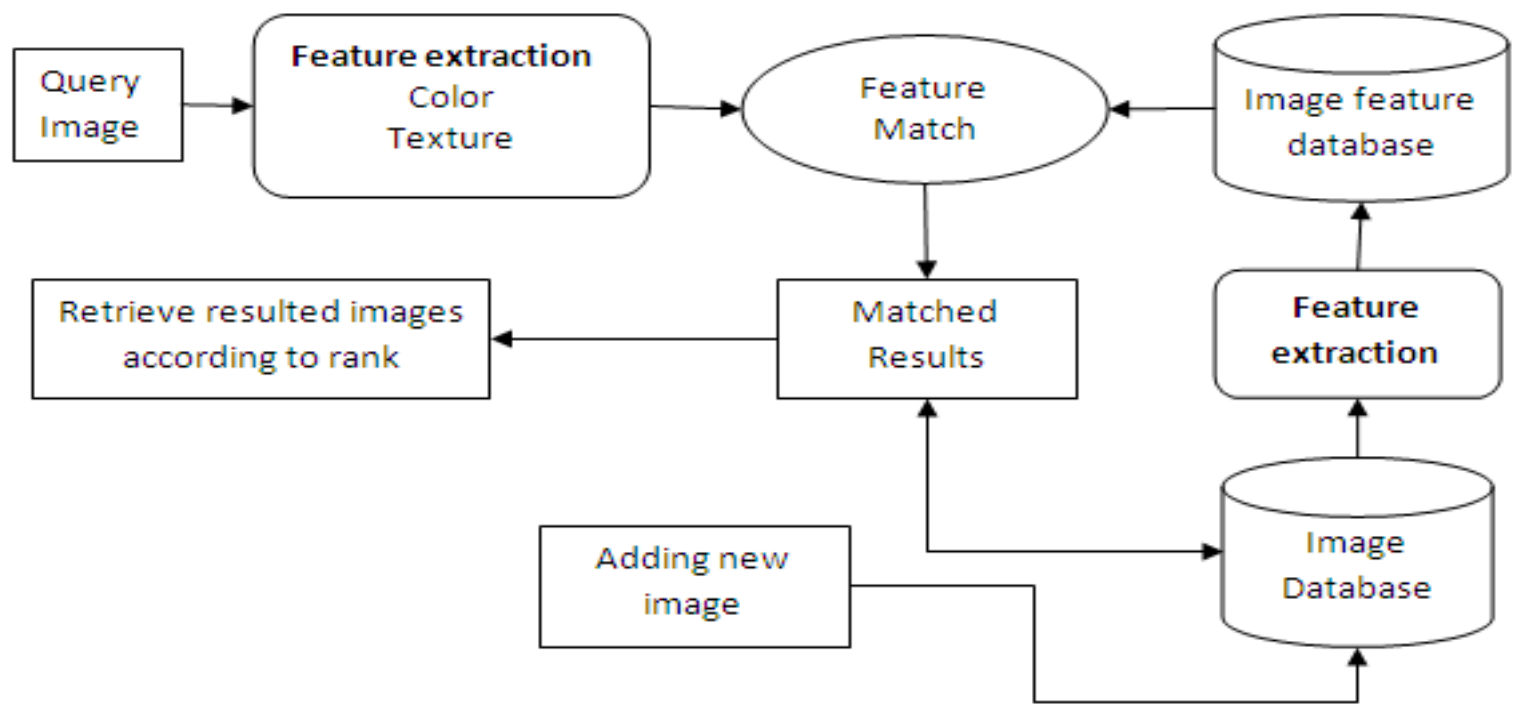

Fig.2. .Basic block diagram for planned work

\section{CBir EXISTING TeChNiQues}

Kekre, Bharadi et al. have introduced Content Based Image Retrieval using Fusion of Gabor Magnitude and Modified Block Truncation Coding [4]. Gabor filters are a group of wavelets, with each wavelet capturing energy at a specific frequency and a specific direction. Expanding a signal using this basis provides a localized frequency description, therefore capturing local features/energy of the signal. Texture features can then be extracted from this group of energy distributions. And modified block truncation is used to retrieve color feature from image. They proved that the proposed system is giving higher Precision and Recall as compared to only Gabor and Only MBTC based CBIR. Gabor feature gives good response to texture of the image and Modified BTC give good response to color content of image.

J. Zhang and W. Zou [5] have presented a novel technique that employs both the color and edge direction features for Content-Based Image Retrieval (CBIR). In this method, a given image is first divided into sub-block which has the same size and then the color and edge direction features of each sub block can be extracted. Next, it constructs a codebook of color feature using clustering algorithm and then each sub-block is mapped to the codebook. The color feature is used to retrieve images, and the edge direction feature is the weight of the similarity measure for the color feature.

Rose and Shah carried on a research project to improve the accuracy of CBIR Using Gradient Projections [6]; the image's structural properties were examined to distinguish one image from another. By examining the specific gray level of an image, a gradient can be computed at each pixel. Pixels with a magnitude larger than the thresholds are assigned a value of 1 . These binary digits are added across the horizontal, vertical, and diagonal directions to compute three projections. These vectors are then compared with the vectors of the image to be matched using the Euclidean Distance Formula. These numbers are then stored in a bookmark so that the image needs only be examined once. A program has been developed for
Matlab that performs this method of projecting gradients. Three databases were amassed for the testing of the proposed system's accuracy: 82 digital camera pictures, 1,000 photographic images, and a set of object orientated photos. The program was tested with $100 \%$ accuracy with all submitted images to the database, and was able to distinguish between pictures that fooled previous CBIR engines. The weakness of this project was its color-blindness.

A CBIR method based on color-spatial feature has been proposed by Lei, Fuzong \& Zhang[ 7]. They proposed a fast algorithm which could include several spatial features of color in an image for retrieval because except for the color histogram information, the position information of each color plays an important role too. These features are area and position, which mean the zero-order and the first-order moments, respectively. By computing the moments of each color region the similarity of two images according to the weight of each factor can be computed. In fact, these features are a kind of representation for image in the scale of low resolution, and the sample image given by a user is usually a draft drawn by hand. Moreover, when a user judges the similarity between two pictures, he will firstly judge them in coarse scale. In this sense, this method is close to the vision model of our eyes. Because the features are simple and can be calculated in fast speed, better result can be made easily through training.

Dr H B Kekre ,S D Thepade et al. introduced Image Retrieval with Shape Features Extracted using Gradient Operators and Slope Magnitude Technique with BTC [9] and tested on generic image database with 1000 images spread across 11 categories. The average precision and recall of all queries are computed and considered for performance analysis. Gradient operators used for shape extraction were Robert, Prewitt, Sobel and Canny which are known as 'MaskShape-BTC' CBIR techniques. The problem with these MaskShape-CBIR methods is the need of resizing the database images to match it with the size of query. This drawback is removed using proposed Mask-Shape-BTC-CBIR methods. In 
proposed image retrieval techniques the feature vectors are formed by applying the block truncation coding (BTC) on the shape image obtained using slope magnitude applied on gradient of the image in both horizontal and vertical direction.

Y.N.Mamatha and A.G. Ananth worked on Content Based Image Retrieval of Satellite Imageries Using Soft Query Based Colour Composite Techniques[14]. They realized that using colours as a content, content based image processing have been carried out for a sample of high resolution urban image and low resolution rural image scenes obtained from satellites.

A research paper on Content-based satellite cloud image retrieval by Deepak Upreti [15] has been developed using gray level, texture and shape as retrieval features from the satellite image repository. The system allows the user to search for an image on the basis of any of the three features alone or in combination by assigning weights to the features. The histogram approach is used to extract the gray level feature, texture feature is extracted using gray level co-occurrence matrix method and the shape feature is extracted using the morphological operations. The images and the extracted feature vectors are stored in the Oracle $10 \mathrm{~g}$ database. Euclidean distance metric is used to compute the similarity between the images. The system is robust as it provides search based on the multiple features. The performance of the system was evaluated by analyzing the retrieval results using precision. The proposed method of image retrieval performs well for the query image. The precision in retrieving the images is high in the proposed method as compare to the other methods of image retrieval.

\section{PROPOSED TECHNIQUE}

As we have discussed in previous section there are many techniques which are introduced to reduce semantic gap and to retrieve similar images. But still scope of improvement is possible. Non-relevant images get retrieved along with relevant images. It is difficult to implement technique which will provide zero non-relevant image retrieval. But we can try to reduce this possibility and may design a technique with maximum relevant images retrieval. To achieve this, here we are going to merge to techniques which are mentioned below. The overview of proposed technique is shown in figure(2).

\section{a. Modified Block Truncation COding}

Block truncation coding (BTC) is a relatively simple image coding technique developed in the early years of digital imaging. This method first divides the image into small nonoverlapping image blocks. The small blocks are coded one at a time. For each block, the original pixels within the block are coded using a binary bitmap the same size as the original block and two mean pixel values[4]. The method first computes the mean pixel value of the whole block and then each pixel in that block is compared to the block mean. If a pixel is greater than or equal to the block mean, the corresponding pixel position of the bitmap will have a value of 1 , otherwise it will have a value of 0 . The simplest extension was to view a color image as consisting of three independent grey scale images and apply BTC to each color plane independently. Most color images are recorded in RGB space, which is perhaps the most well-known color space [11].
In modified BTC to create a binary bitmap in the RGB space, an inter-band average image (IBAI) is first created and a single scalar value is found as the threshold value.

First we will resize the image in $256 * 256$ pixels. Now let $X=\{r(i, j), g(i, j), b(i, j) i=1,2, \ldots . m . j=1,2, \ldots \ldots n\}$ be an $m x n$ color block in RGB space where $\mathrm{m}=\mathrm{n}=256$.

$i_{b}=\frac{1}{3} r(i, j)+g(i, j)+b(i, j) \ldots .(1)$

The threshold is computed as the mean of each color and using this threshold binary of bitmap can be calculated[12] as shown in figure(3).

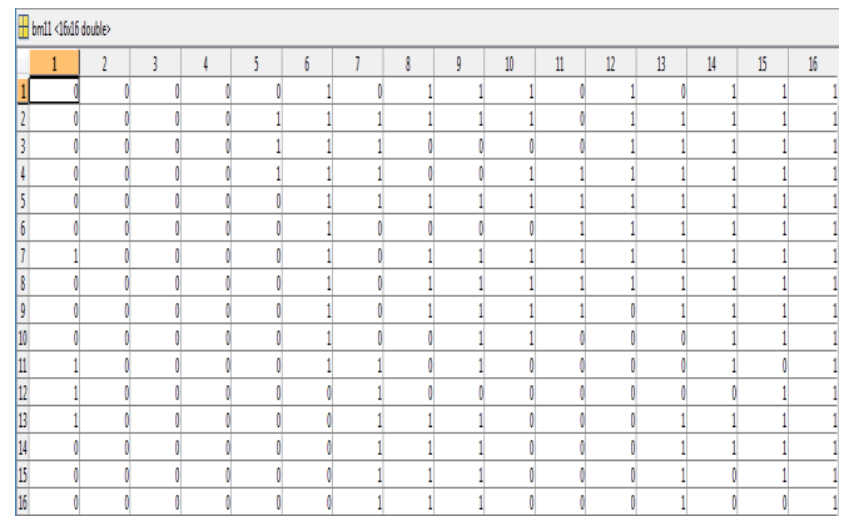

Fig.3. Bitmap of image

After the creation of the bitmap, two representative (mean) colors are then computed. The two mean colors are $\mathrm{MC} 1$ $=\{\mathrm{Cr} 1, \mathrm{Cg} 1, \mathrm{Cb} 1\}$ and $\mathrm{MC} 2=\{\mathrm{Cr} 2, \mathrm{Cg} 2, \mathrm{Cb} 2\}$. Where MC1 represents uppermean components and $\mathrm{MC} 2$ represents lowermean components[12].

\section{b. PATtern Generation FOR TEXTURE}

Patterns are generated for extracting texture feature of image. Each pattern defines different formats of textue. The idea is to map the image equivalent to patterns and then the different texture pattern '16-pattern' generated using Kekre's transform matrix.

Number of patterns can be generated using transform matrices namely 4 pattern, 16 pattern, 64 pattern. NxN matrix can be used to generate $\mathrm{N} 2$ patterns. For example, if we want to generate 16 pattern then $4 \times 4$ matrix need to be used. Element wise multiplication of each row of the transform matrix is taken with all possible rows of the same matrix.

The 16 Kekre texture patterns [13] is generated using Kekre transform matrices of size $4 \times 4$. Figure 4 gives $2 \times 2$ kekre matrix and generation of four Kekre texture patterns. $2 \times 2$ Kekre transform matrix is shown in figure, each row of this matrix is considered one at a time and is multiplied with all rows of the same matrix to generate Kekre texture patterns as shown in figure 5. The $4 \times 4$ Kekre transform matrix is given in figure 6 and visualization of 16 Kekre transform patterns generated using it is shown in figure 8 , where black and grey colour scaled between 1 to 256 in the pattern and 0 is represented by white colour. 


$$
\left[\begin{array}{cc}
1 & 1 \\
-1 & 1
\end{array}\right]
$$

Fig.4. 2x2 Kekre Matrix
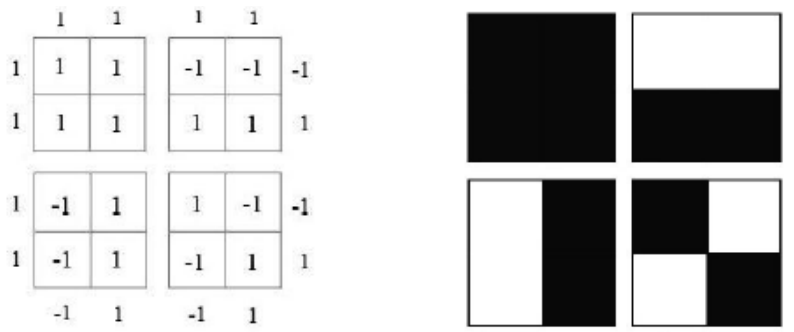

Fig.5. 4-Kekre Pattern Generation

$$
\left[\begin{array}{cccc}
1 & 1 & 1 & 1 \\
-3 & 1 & 1 & 1 \\
0 & -2 & 1 & 1 \\
0 & 0 & -1 & 1
\end{array}\right]
$$

Fig.6. $\quad 4 \times 4$ Kekre Matrix

\section{c. Fusion OF MBTC \& PATtERNS FOR SATEllite IMAGE RETRIEVAL}

Satellite image consist of different classes are stored in in hard disk. These images are divided into 256x256 pixel size blocks passed through feature vector extraction module and resultant feature vector is again stored as a MAT file [4].

\section{A. Feature Extraction Module}

i. Any query image or block of satellite image will be first divided into $4 \times 4$ pixel block and MBTC technique is applied on each block. MBTC basically separates each block into three components i.e. red, green and blue. For each component Uppermean and Lowermean is calculated. It results into six components separated from one image i.e. uppermean red $(\mathrm{Cr} 1)$, uppermean green $(\mathrm{Cg} 1)$, uppermean blue (Cb1) and lowermean red (Cr2), lowermean $(\mathrm{Cg} 2)$, lowermean blue (Cb2) [4],[5].

ii. We calculated 16 grey patterns using $4 \times 4$ Kekre's transform matrix i.e. P1, P2,P3,P4,P5,P6 and so on

iii. Then we will check for the grey levels existing in Kekre's pattern and quantize six components calculated by step (i) into these grey levels. Here we will get quantized grey components.

iv. Now to form a feature vector, each pattern calculated at step (ii) will be superimposed on $\mathrm{Cr} 1, \mathrm{Cg} 1, \mathrm{Cb} 1, \mathrm{Cr} 2, \mathrm{Cg} 2$,
$\mathrm{Cb} 2$. E.g. first we will superimpose $\mathrm{P} 1$ with $\mathrm{Cr} 1$ (as shown in figure 4.14) and check for how many times P1 exist in $\mathrm{Cr} 1$ (or count of P1 present in Cr1). This count will be stored as the first element of one matrix.

v. Step (iv) is repeated to find P2 to P16 count in $\mathrm{Cg} 1, \mathrm{Cb} 1$, $\mathrm{Cr} 2, \mathrm{Cg} 2$ and $\mathrm{Cb} 2$ components of the image.

vi. Step (iv) \& (v) will be resulted into $12 \times 8$ matrix which is declared and stored as a 'feature vector'.

When Query object image is fired, it is forwarded to feature vector extraction module and feature vector also calculated for it. Then satellite image (figure-4.15) resized in multiple of 256 and divided into blocks of 256x256 pixel size and each block is passed through feature extraction module. These extracted features for each blocks are stored in different matrices.

Feature vector of query object image is compared with feature vector of satellite image blocks stored in database goes through similarity measurement. Here we compared feature vectors using absolute distance. According to distance measured between query object and blocks of satellite image blocks, similar blocks are retrieved.

\section{RESUlt AND Discussion}

For each image red, green and blue components are separated from uppermean and lowermean as shown in figure 7 and quantized in pattern values as shown in figure 8 ,
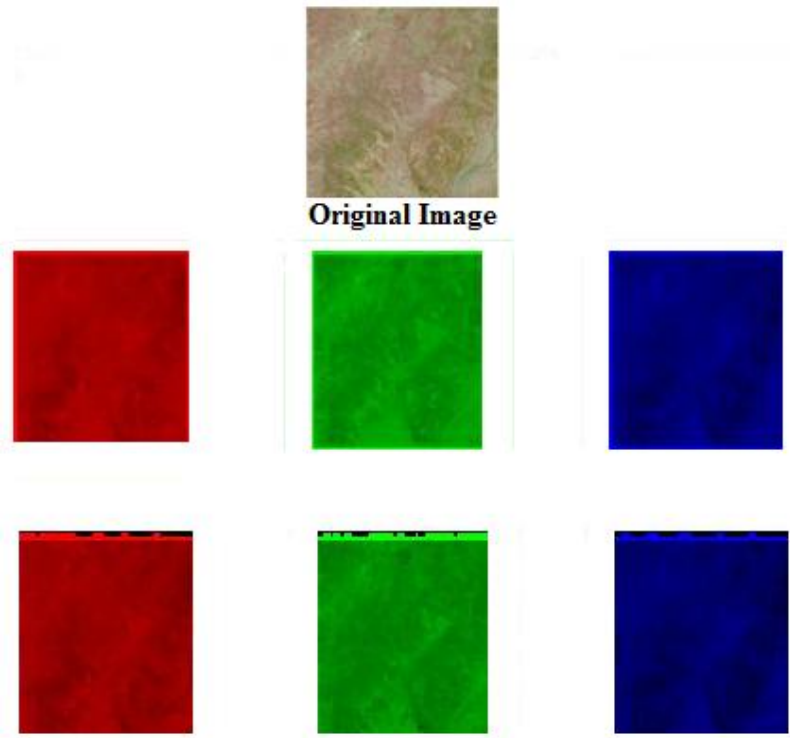

Fig.7. Upperman and lowermean for R,G,B components 

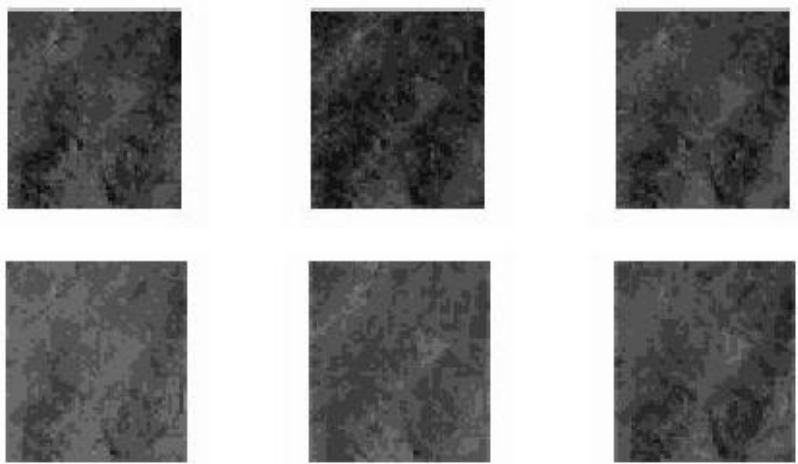

Fig.8. Quantized upperman and lowermean matrix

Using $4 \times 4$ Kekre matrix 16-pattern transform generated as shown in figure 9.
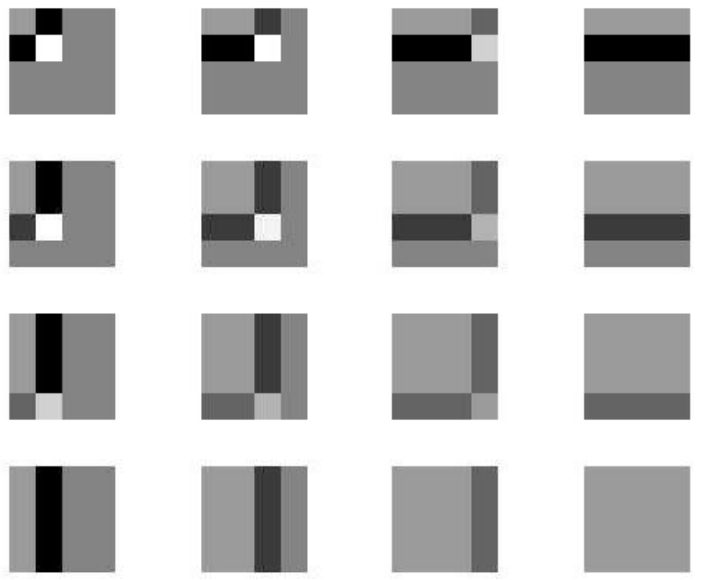

Fig.9. 16-Kekre Pattern Generation

Find co-occurrence of each pattern shown in figure 9 is checked with components in figure 8. This co-occurrence matrix is nothing but feature vector as show in figure 10,

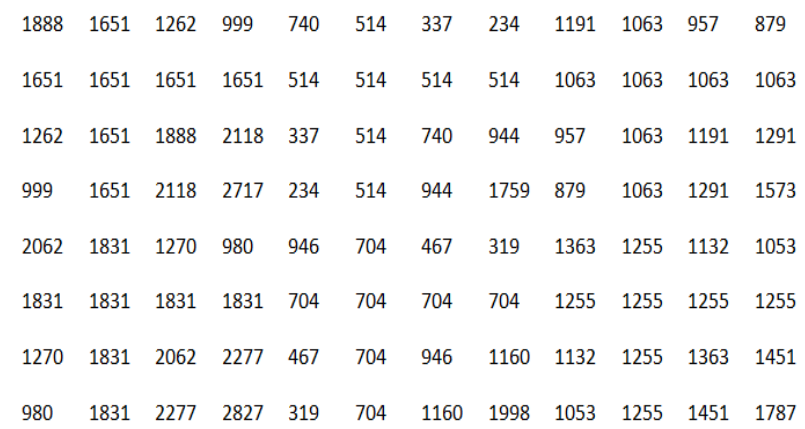

Fig.10. (8x12) feature vector

A satellite image is divided into blocks of 256x256 pixel size as shown in figure 11.
International Conference \& Workshop On Advance Computing 2013

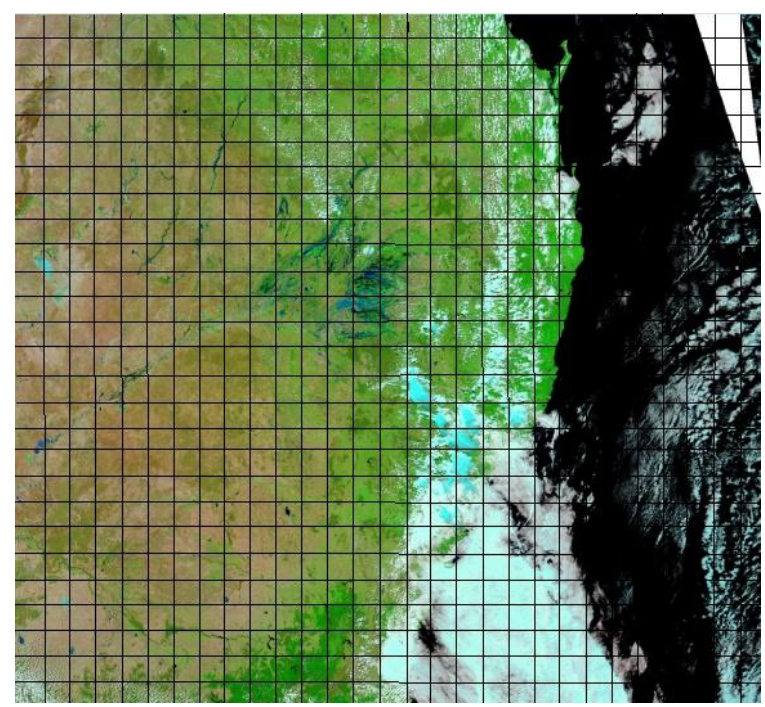

Fig.11. Original Satellite image of size [6800x6400] resized into multiple of 256 and divided into blocks of (256x256) pixel size

Now we will apply fusion based Content based image retrieval technique on each block and save feature vector of each block on hard disk. Each block is of (256x256) pixel size and after applying discussed technique on each block we will get feature vector of $(8 \times 12)$ size for each block. For this image we got 675 blocks of size (256x256) pixel on which we had applied CBIR technique and stored in a MAT file.

Then we had calculated FV for query object which is sample picture (size 256x256) of sand and greenery portion shown in figure 7 .

Now to test the retrieval technique,we had segregated five query object block from original satellite image as shown in figure 12.

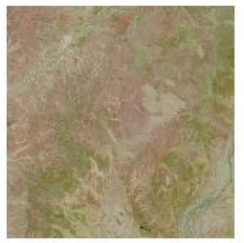

Block 1

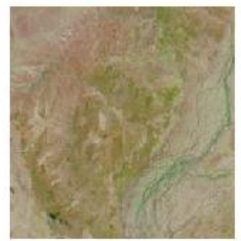

Block 2

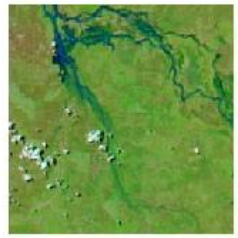

Block 3

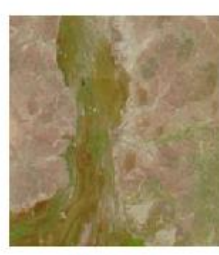

Block 4

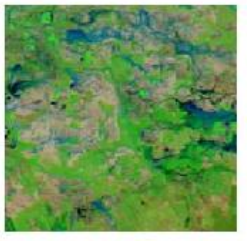

Block 5
Fig.12. Segregated blocks from original image

Now we will fire each block as a query object to find out similar objects from the satellite image shown in figure 11 . Retrieval results are shown below from figure 13 to figure 17. Relevant blocks to the query object are highlighted in results below. 


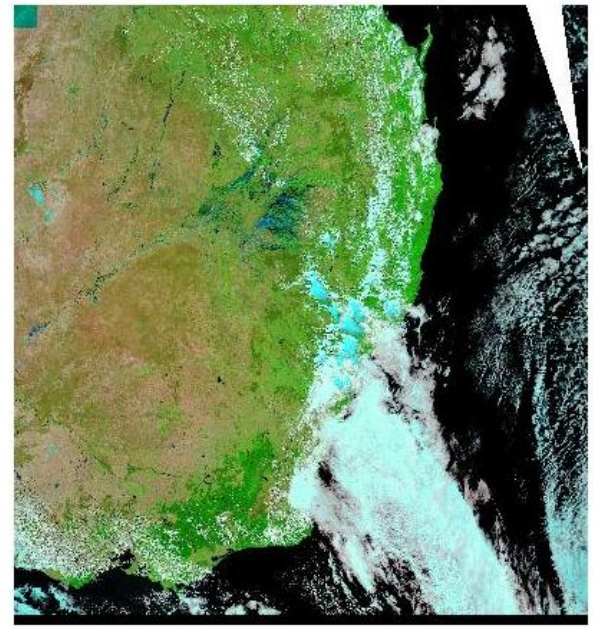

Fig.13. Retrieval output for block 1 from image 1

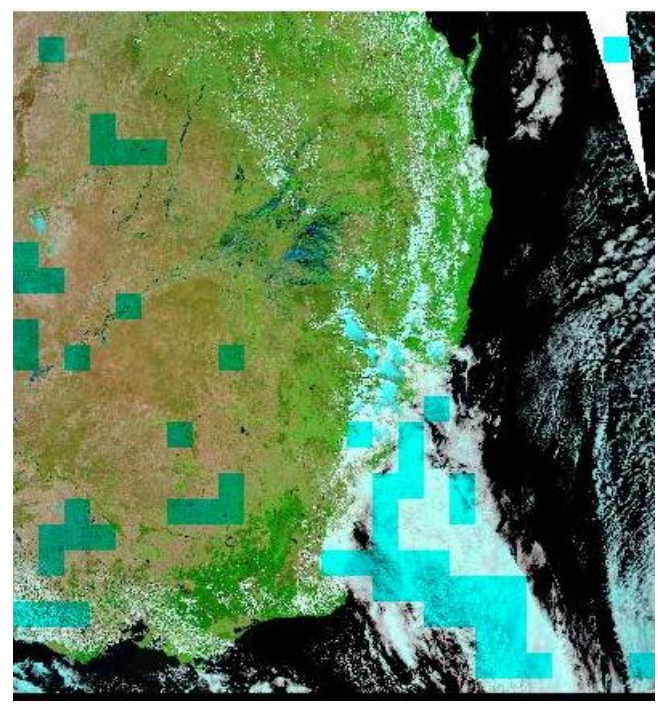

Fig.14. Retrieval output for block 2 from image 1

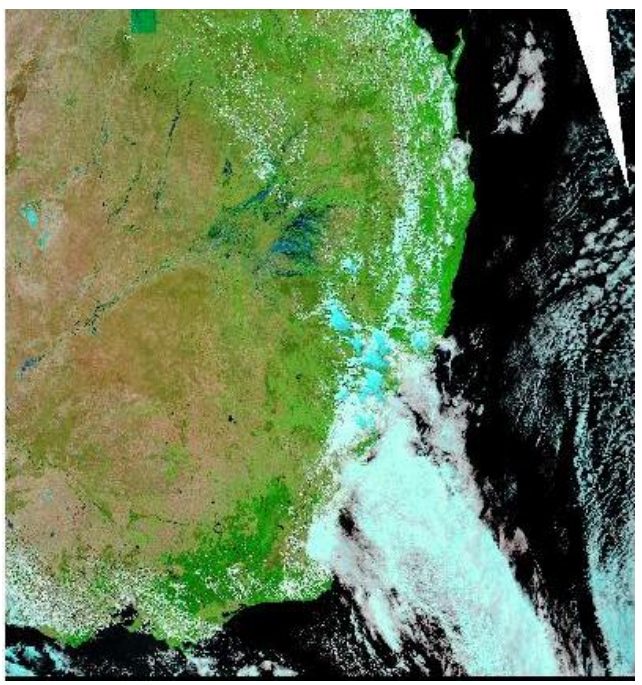

Fig.15. . Retrieval output for block 3 from image 1

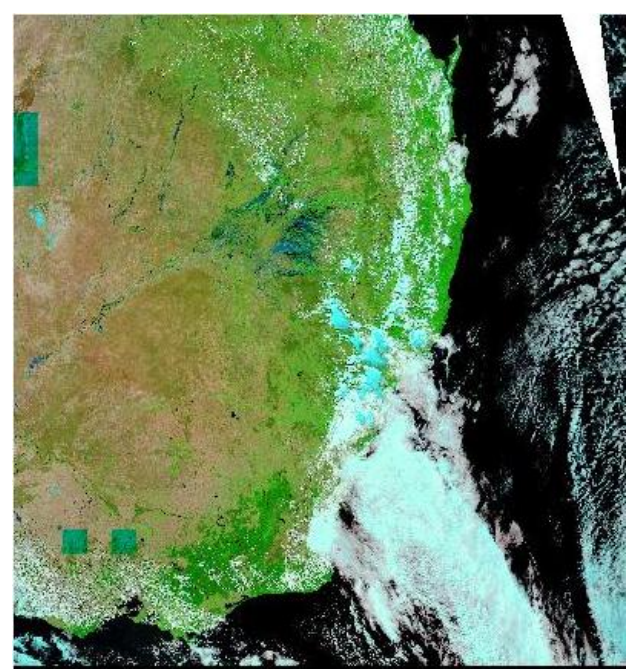

Fig.16. Retrieval output for block 4 from image 1

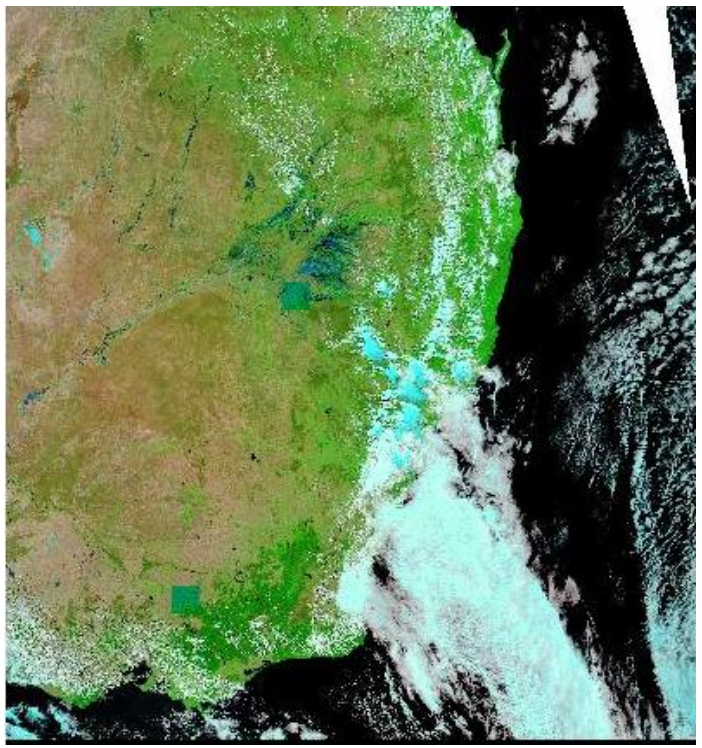

Fig.17. Retrieval output for block 5 from image 1 
TABLE I. TEST RESUlts OF SATELlite IMAGE RETRIEVAL

\begin{tabular}{|c|c|c|c|c|c|c|}
\hline $\begin{array}{c}\text { Satellite } \\
\text { Images }\end{array}$ & Block No. & $\begin{array}{c}\text { Total } \\
(256 \times 256) \\
\text { Blocks in } \\
\text { Images }\end{array}$ & $\begin{array}{c}\text { Retrieved } \\
\text { Relevant } \\
\text { Blocks }\end{array}$ & $\begin{array}{c}\text { Query Block } \\
\text { Retrieved or not }\end{array}$ & $\begin{array}{c}\text { Probability } \\
\text { of block } \\
\text { Retrieval }\end{array}$ & $\begin{array}{c}\text { Efficiency of } \\
\text { Retrieval } \\
(\%)\end{array}$ \\
\hline \multirow{5}{*}{ Image 1} & 1 & 675 & 1 & Yes & 1 & 100 \\
\hline & 2 & 675 & 103 & Yes & 0.01 & 1 \\
\hline & 3 & 675 & 1 & Yes & 1 & 100 \\
\hline & 4 & 675 & 5 & Yes & 0.20 & 20 \\
\hline & 5 & 675 & 2 & Yes & 0.50 & 50 \\
\hline \multirow{5}{*}{ Image 2} & 1 & 64 & 1 & Yes & 1.00 & 100 \\
\hline & 2 & 64 & 1 & Yes & 1.00 & 100 \\
\hline & 3 & 64 & 2 & Yes & 0.50 & 50 \\
\hline & 4 & 64 & 2 & Yes & 0.50 & 50 \\
\hline & 5 & 64 & 1 & Yes & 1.00 & 100 \\
\hline \multirow{5}{*}{ Image 3} & 1 & 864 & 13 & Yes & 0.08 & 8 \\
\hline & 2 & 864 & 2 & Yes & 0.50 & 50 \\
\hline & 3 & 864 & 138 & Yes & 0.01 & 1 \\
\hline & 4 & 864 & 1 & Yes & 1.00 & 100 \\
\hline & 5 & 864 & 3 & Yes & 0.33 & 33 \\
\hline \multirow{5}{*}{ Image 4} & 1 & 168 & 6 & Yes & 0.17 & 17 \\
\hline & 2 & 168 & 2 & Yes & 0.50 & 50 \\
\hline & 3 & 168 & 20 & Yes & 0.05 & 5 \\
\hline & 4 & 168 & 1 & Yes & 1.00 & 100 \\
\hline & 5 & 168 & 1 & Yes & 1.00 & 100 \\
\hline \multirow{5}{*}{ Image 5} & 1 & 360 & 41 & Yes & 0.02 & 2 \\
\hline & 2 & 360 & 1 & Yes & 1.00 & 100 \\
\hline & 3 & 360 & 2 & Yes & 0.50 & 50 \\
\hline & 4 & 360 & 1 & Yes & 1.00 & 100 \\
\hline & 5 & 360 & 6 & Yes & 0.17 & 17 \\
\hline
\end{tabular}

Similar test has been performed on other four satellite images. Five block queries are segregated from other satellite images and retrieved from each image. Retrieval results for each image is as shown in table I. From the results we can conclude that for all images we had retrieved relevant blocks using the implemented retrieval technique. But efficiency for each test is different. Analysis of efficiency of retrieval we achieved is as shown in table 2 .

Table II - Efficiency of retrieval

\begin{tabular}{|l|l|l|}
\hline Min. efficiency & Max. efficiency & Avg. efficiency \\
\hline $1 \%$ & $100 \%$ & $52.16 \%$ \\
\hline
\end{tabular}

Analysis shows that average efficiency for implement technique is greater than $50 \%$.

From table 1, we can observe that there are 2 test which are completely failed where we got efficiency of $1 \%$.

Therefore, $(2 / 25) * 100=8 \%$ of the tests are unsuccessful. Six tests are below $33 \%$ efficiency are partially successful i.e. $(6 / 25) * 100=24 \%$ of the total test are giving average retrieval results. And 15 out of 25 tests had achieved the efficiency above $50 \%$ i.e. $(15 / 25)^{*} 100=60 \%$ tests of the total tests are able to retrieve highly localized area.

\section{CONCLUSION}

In this paper we have proposed a satellite Image Retrieval technique using Color \& texture features. This technique is 
based on MBTC \& Kekre Transform Patterns. This method is used for specific region block extraction from the high resolution Satellite image. Max retrieval accuracy was $100 \%$, out 25 tests $60 \%$ tests were giving accuracy above $50 \%$ and high localization of the query block. This technique is very much suitable for searching a particular region from the satellite image.

\section{REFERENCES}

[1] W.M. Smeulders,"Content-Based Image Retrieval at the End of the Early Years",IEEE TRANSACTIONS ON PATTERN ANALYSIS AND MACHINE INTELLIGENCE, VOL. 22, NO. 12, DECEMBER 2000

[2] G. Rafiee, S.S. Dlay, and W.L. Woo,"A Review of Content-Based Image Retrieval",IEEE, 2010. Tavel, P. 2007 Modeling and Simulation Design. AK Peters Ltd.

[3] Y N Mamatha and A.G Ananths and S O Neil, "Content Based Image Retrieval of Satellite Imageries Using Soft Query Based Color Composite Techniques",", IEEE Trans on Acoustic speech signal processing, Vol 1, No.3, pp. 1278-1288, 1986

[4] Kekre H.B., Bharadi, V.A., Thepade S.D., Mishra B.K., Ghosalkar, S.E., Sawant S.M. , "Content Based Image Retrieval Using Fusion of Gabor Magnitude and Modified Block Truncation Coding", IEEE computer society, 2010 IEEE

[5] J. Zhang and W. Zou, "Content-Based Image Retrieval Using Color and Edge Direction Features",2010 IEEE

[6] Jed Rose and Mubarak Shah,"Content-Based Image Retrieval Using Gradient Projections",1998 IEEE

[7] Zhang Lei, Lin Fuzong, Zhang Bo,"A CBIR method based on colorspatial feature",1999 IEEEE
[8] Zhao Hai-ying, Xu Zheng-guang, Penghong,"A Texture Feature Extraction Based On Two Fractal Dimensions for Content_based Image Retrieval",2008 IEEE

[9] Dr.H.B.Kekre, S.D. Thepade et al.,"Image Retrieval with Shape Features Extracted using Gradient Operators and Slope Magnitude Technique with BTC",International Journal of Computer Applications (0975 8887) Volume 6- No.8, September 2010

[10] Dr.H.B.Kekre, S. D. Thepade,"Image Retrieval using Color-Texture Features Extracted from Walshlet Pyramid",ICGST - GVIP Journal, ISSN: 1687-398X, Volume 10, Issue 1, February 2010

[11] H B Kekre and V A Bharadi, "Modified BTC \& Walsh coefficients Based Features for Content Based Image Retrieval" NCICT, India.

[12] A R Sawant, Dr. V A Bharadi, Dr. H B Kekre

B. Markarkandy "Color \&Texture Based Image Retrieval using Fusion of Modified Block Truncation Coding (MBTC) and Kekre Transform Patterns", IJACSA Special Issue on Selected Papers from International Conference \& Workshop On Emerging Trends In Technology 2012

[13] Dr.H.B.Kekre, S.D. Thepade et al.,"Performance Comparison of Gradient Mask Texture Based Image Retrieval Techniques using Walsh, Haar and Kekre Transforms with Image Maps",International Conference on Technology Systems and Management (ICTSM) 2011

[14] Y N Mamatha and A.G Ananths and S O Neil, "Content Based Image Retrieval of Satellite Imageries Using Soft Query Based Color Composite Techniques",", IEEE Trans on Acoustic speech signal processing, Vol 1, No.3, pp. 1278- 1288, 1986

[15] Deepak Upreti, Dr. Sameer Saran, Dr. Nicholas Hamm, "CONTENTBASED SATELLITE CLOUD IMAGE RETRIEVAL", Thesis submitted to the Faculty of Geo-Information Science and Earth Observation of the University of Twente 